\title{
Integration and characterization of the cryogenic system of MEGARA
}

\author{
Ferrusca R.,D.*a, Cisneros G., M. E. ${ }^{\text {a }}$, Velázquez, M. ${ }^{\mathrm{a}}$, Zenteno H., J. A. ${ }^{\mathrm{a}}$, \\ Gil de Paz, A. ${ }^{\mathrm{b}}$, Gallego, J. ${ }^{\mathrm{b}}$, Carrasco, E. ${ }^{\mathrm{a}}$, Sánchez-Moreno F.C. ${ }^{\mathrm{c}}$, Iglesias-Páramo $^{\mathrm{d}}$ \\ \& MEGARA Team \\ ${ }^{a}$ Instituto Nacional de Astrofísica, Óptica y Electrónica, Luis Enrique Erro 1, Sta. Ma. Tonantzintla, \\ Puebla, C.P. 72840, Mexico; \\ ${ }^{b}$ Universidad Complutense de Madrid; ${ }^{\circ}$ Universidad Politécnica de Madrid; \\ ${ }^{\mathrm{d}}$ Instituto de Astrofísica de Andalucía.
}

\begin{abstract}
MEGARA (Multi-Espectrógrafo en GTC de Alta Resolución para Astronomía) is an optical Integral-Field Unit and Multi-Object Spectrograph designed for the GTC (Gran Telescopio de Canarias) 10.4m telescope in La Palma, it is expected that the spectrograph will be delivered to GTC towards the end of 2016. MEGARA includes an open cycle cryostat which harbors the scientific CCD of the instrument at an operating temperature of $153 \mathrm{~K}$, this cryogenic system has been designed and integrated by the "Astronomical Instrumentation Lab for Millimeter Wavelengths" at the Instituto Nacional de Astrofísica, Óptica y Electrónica (INAOE) in Mexico. Early this year the cryostat has finished its fabrication and now it is on AIV phases, in this paper we report the cryostat CCD-head and dewar back integration, vacuum and cryogenic test results are also reported. The final integration of the cryostat with the other components of the instrument is taking place at LICA lab at the Universidad Complutense de Madrid.
\end{abstract}

Keywords: MEGARA, open-cycle cryostat, cryostat, GTC, Integral-Field Unit Spectrograph, Multi-Object Spectrograph.

\section{INTRODUCTION}

MEGARA (Multi-Espectrógrafo en GTC de Alta Resolución para Astronomía) ${ }^{1,2,3}$ is a consortium integrated by the Universidad Complutense de Madrid (UCM, Spain), the Instituto Nacional de Astrofísica, Óptica y Electrónica (INAOE, México), the Instituto de Astrofísica de Andalucía (IAA-CSIC, Spain), and the Universidad Politécnica de Madrid (UPM, Spain). The instrument is designed for the $10.4 \mathrm{~m}$ GTC telescope in La Palma (Spain) that will be installed on the telescope by the end of 2016. MEGARA will offer two observing modes: a Large Compact Bundle (LCB) which is part of an Integral Field Unit (IFU) to cover a region of 12.5 " x 11.3" with an spaxel of 0.62 arcsec, and a Multi-Object Spectroscopy (MOS) mode that will be able to observe up to 100 objects on $3.5^{\prime}$ x $3.5^{\prime}$ field around the LCB. Both the LCB IFU and MOS capabilities of MEGARA will provide intermediate-to-high spectral resolutions (R 6000,11000 and 18700 ) in the wavelength range of $3650-10000 \AA$.

The spectrograph is a refractive optical system integrated by a pseudo-slit and a shutter to receive the incoming radiation from the optical fibers, afterwards is located the collimator-camera system that includes a total of 11 Volume Phase Holographic gratings (VPHs) to provide the different spectral resolutions of the instrument, the VPHs are placed in the pupil by means of a wheel and an insertion mechanism. Finally at the optical exit of the camera is placed the custommade cryostat that harbors an E2V231-84 4kx4k CCD.

\section{CRYOSTAT INTEGRATION}

MEGARA cryostat ${ }^{4,5}$ has finished its fabrication according to the detailed design and has been integrated and tested at INAOE instrumentation laboratories before being shipped to LICA laboratory at UCM for its final integration with the other subsystems of the instrument. The cryostat has been tested with the engineering CCD at LICA, which will ultimately be replaced by the scientific CCD.

The selected cryogenic device to harbor the CCD for the MEGARA spectrograph is a liquid nitrogen open-cycle cryostat and by requirement is designed to be in an static horizontal mounting on an optical table. The complete cryostat assembly consists of two main parts: the Dewar Back and the CCD Head, which are assembled together with temperature and pressure sub-systems. The conceptual, preliminary and detailed designs of this cryostat are described

Ground-based and Airborne Instrumentation for Astronomy VI, edited by Christopher J. Evans, Luc Simard, Hideki Takami Proc. of SPIE Vol. 9908, 99087Y · (c) 2016 SPIE · CCC code: 0277-786X/16/\$18 · doi: 10.1117/12.2231797 
elsewhere ${ }^{6,7,8}$. In the next sections we report the critical parts of the assembly of the cryostat as well as the results obtained under vacuum and cryogenic conditions.
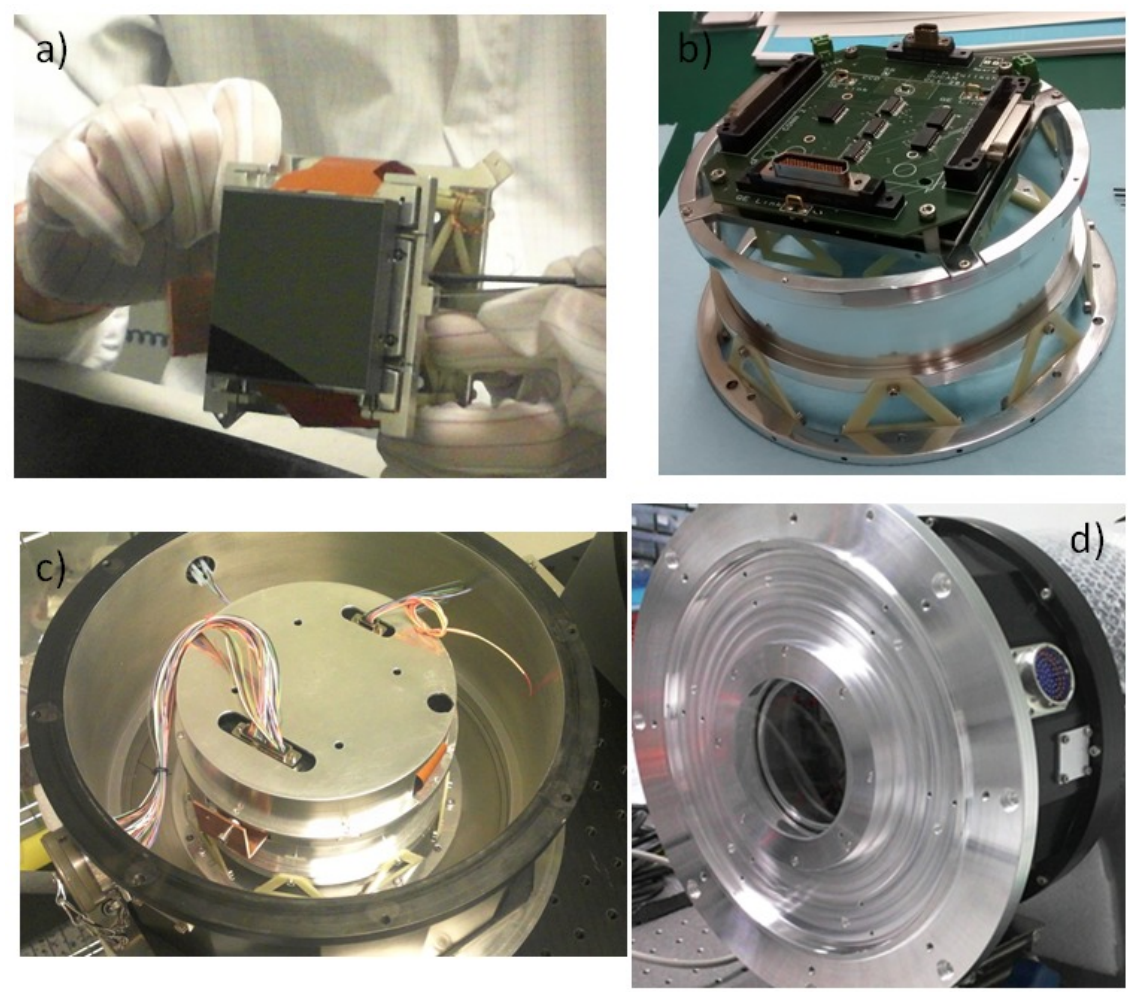

Figure 1, CCD head views of MEGARA cryostat: a) CCD 231-84 from E2V mounted on kinematic support and being adjusted for its final position, operating temperature is close to $153 \mathrm{~K}$ and is regulated by a Lakeshore 335 and a $51 \mathrm{Ohm}$ heater. b) PCB mounted on support structure of the CCD radiation shield, operating temperature is $250 \mathrm{~K}$. c) CCD radiation shield completely assembled and mounted on vacuum shroud, cables from CCD and PCB are connected to hermetic connectors and copper thermal link can be seen coming out from the shield to be connected to cold finger on dewar back. d) CCD head completely assembled with front flange with CAM-S7 lens sealing the cryostat window.

\subsection{CCD head assembly}

CCD Head part contains the CCD and its associated electronics ${ }^{4}$, and it provides two electrical ports to read-out the signals from the CCD and temperature control and monitoring. A front flange lid contains the last lens (CAM-S7) of the MEGARA spectrograph which also serves as a vacuum window. The CCD and its mountings are surrounded by an aluminum radiation shield that will help to improve the hold-time of the cryostat. CCD Head consists of five main parts: the CCD mounting, the CCD and PCB supporting structure, the CCD housing, the CCD head main body and lens CAMS7.

The CCD mounting is a baseplate to mount the CCD and maintain it adjusted in the correct position according to the optical axis of the spectrograph and it allows to correct in X, Y, Z, tip and tilt the position if it is necessary by means of a custom made kinematic mounting which allows movements as low as $20 \mathrm{um}$. This component includes in the back a 51 $\mathrm{Ohm}$ heater and a temperature sensor, which are connected to a Lakeshore 335 to control the detector temperature by a PID control. The mounting is also designed to provide connectivity to an OFHC copper thermal link to the LN2 tank.

The CCD and PCB supporting structure is a G10 structure that allows mounting the CCD mounting and the CCD PCB, it also has a radiation shield in between to minimize radiation to the detector area. The PCB is isolated from the structure with nylon supports. CCD heater and CCD temperature sensor have copper wires long enough $(200 \mathrm{~mm})$ to reduce the heat conduction between the CCD mounting and the PCB. 
The CCD housing holds the CCD and PCB supporting structure and keeps its components thermally isolated from the CCD Head front lid flange by G10 supports, it also includes a radiation shield that surrounds the mentioned components and several holes to provide access to the adjusting screws of the CCD mounting support and exit for the cabling to external connectors.
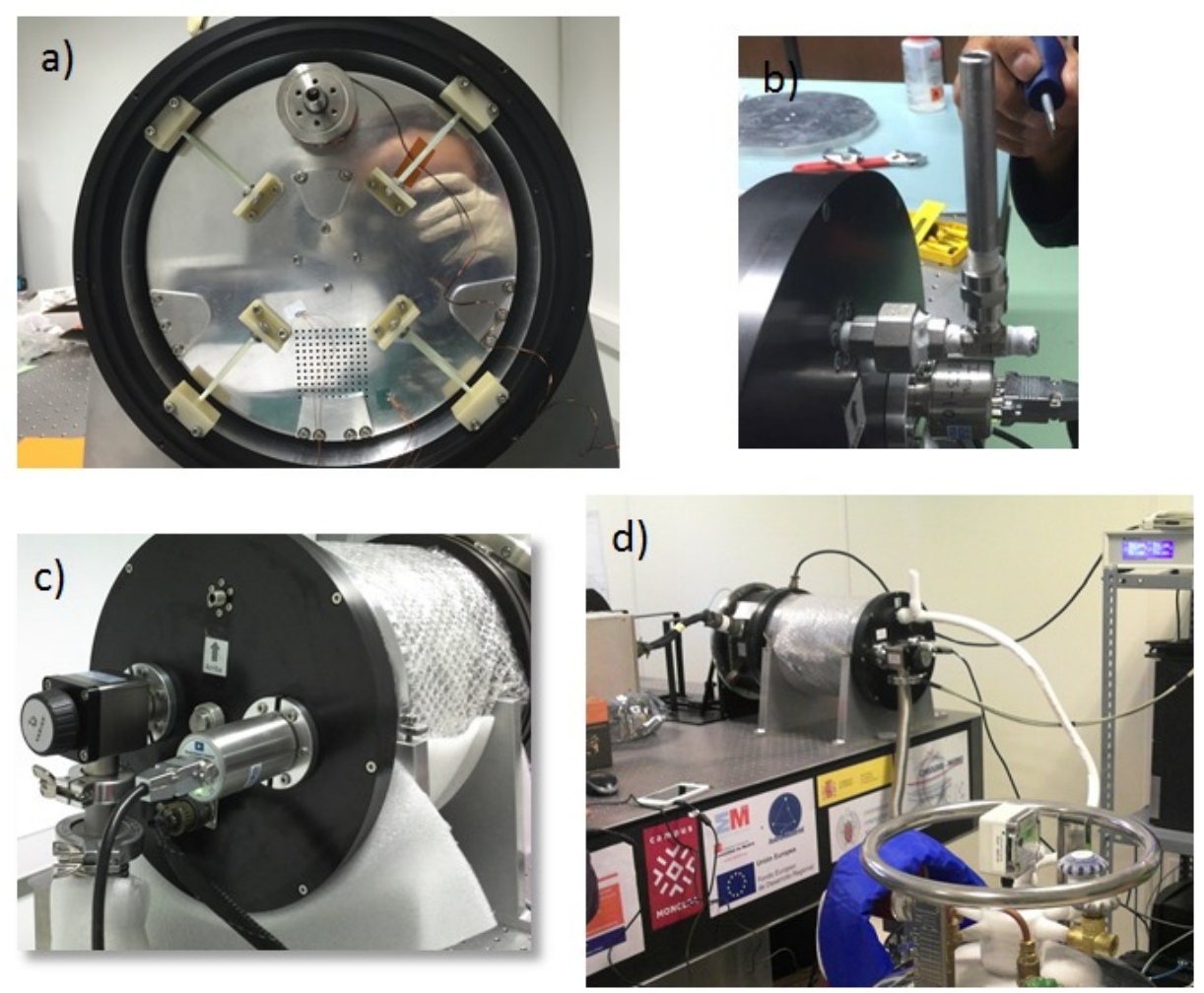

Figure 2, Dewar back views of MEGARA cryostat: a) Internal assembly on dewar back showing the radiation shield and its isolating G10 supports, LN2 tank and cryopump are inside. b) Assembly of LN2 filling tool on the exit of the filling tube. c) Dewar back showing (from top center and clockwise) filling tube exit, pressure gauge, temperature cable, vacuum valve and over pressure relief valve (center). d) Complete assembly of cryostat on optical table showing: cryostat CCD head connected to CCD controller (left) and temperature controller (up right); dewar back connected to Lakeshore and pressure monitor (not visible) and filling tube during a LN2 transference.

Finally the CCD head main body is a vacuum shroud with a front flange which is used as surface reference when the cryostat is coupled to the camera of the spectrograph, it also has two electrical ports by means of hermetic connectors, the entrance of radiation is through CAM-S7 the last lens of the camera which is installed in the flange by using a custom mounting with o-ring to provide the necessary vacuum conditions to operate the cryostat. There is also a lateral plate which provides access to the internal thermal link. Figure 1, provides several views of the described components.

\subsection{Dewar back assembly}

The dewar back contains the liquid nitrogen (LN2) tank which is surrounded by an aluminum radiation shield; it also has on the rear part the liquid nitrogen fill tube, an electrical port for temperature monitor, a vacuum port for turbo pump and a vacuum port for pressure gauge, and a safety valve relief.

MEGARA cryostat was designed to operate in horizontal position, so the LN2 tank support design has been made by means of the use of G10 rods which maintain it concentrically with respect to the vacuum jacket, the radiation shield is supported in a similar way.

On the top part of the SS316 LN2 tank is attached a copper cold finger with a temperature sensor and on the back of the tank is a cryopump which includes a $10 \mathrm{Ohms} / 20 \mathrm{~W}$ heater and a temperature sensor. Sensors and heater are wired to 
the back of the dewar back with copper cable long enough to minimize thermal contributions from the outside. On the back of the tank there is welded filling tube which has on its way a clamp that is attached to the radiation shield and includes a temperature sensor. LN2 tank is wrapped with 5 sheets of Mylar superinsulation to improve its hold time. For a LN2 filling it has been designed a custom tool which allows in the outside of the cryostat to be connected with standard LN2 dewars.

\section{PRESSURE AND TEMPERATURE COMPONENTS}

Characterization has been performed on critical components that allow control and monitoring the CCD head and dewar back of the MEGARA cryostat. In the next sub-sections we summarize these results.

\subsection{Temperature sensors and heaters}

The cryostat includes five resistive platinum sensors (PT-103 ${ }^{12}$ ) which have excellent performance on our operating temperature range; the sensors are located on key parts to monitor the cryostat: LN2 filling tube; copper cold finger; CCD mounting support; CCD PCB; and activated charcoal cryopump, used only during maintenance mode. Temperature sensors are wired with AWG 29 copper cables which contribute to the total resistance, therefore the sensors were characterized on a closed cycle cryostat, the temperature offsets might be subtracted by software. The results obtained are shown in table 1.

Table 1. Temperature sensors wiring characterization results.

\begin{tabular}{|c|c|c|c|c|}
\hline Location Sensor & $\begin{array}{c}\text { Wire } \\
\text { Length }(\mathbf{m m})\end{array}$ & $\begin{array}{c}\text { Wire } \\
\text { Resistance }(\Omega)\end{array}$ & $\begin{array}{c}\text { Resistance } \\
\text { Offset }(\Omega)\end{array}$ & $\begin{array}{c}\text { Temperature } \\
\text { Offset }\left({ }^{\circ} \mathbf{C}\right)\end{array}$ \\
\hline LN2 filling tube & 300 & 0.1 & 0.47 & 1.2 \\
\hline Cryopump & 300 & 0.1 & 0.47 & 1.2 \\
\hline Copper cold finger & 2000 & 0.5 & 1.30 & 3.4 \\
\hline CCD mounting support & 200 & 0.1 & 0.00 & 0.0 \\
\hline
\end{tabular}

A $51 \mathrm{Ohm}$ heater is placed on the CCD mounting to provide heating power (up to 2 Watts) to the CCD and regulate its temperature to 153 Kelvin by means of a Lakeshore 335 with a PID control. A second $10 \mathrm{Ohm}(20 \mathrm{~W})$ resistor is placed on the cryopump to provide heat $\left(70-100^{\circ} \mathrm{C}\right)$ and is used only when the cryostat is in maintenance mode to outgas the charcoal.

\subsection{Pressure system}

The pressure sensor used in the cryostat is the 972 DualMag $^{13}$ transducer from MKS which has incorporated cold cathode inverted magnetron and a MicroPirani thermal conductivity MEMS vacuum sensor technologies. One of the requirements that we have to face is that the electronics cabinet of the cryostat will be located at 15 meters from the instrument on the telescope platform; however this sensor as indicated by the manufacturer and technical service has not been tested for cabling longer than $7.5 \mathrm{mts}$. We have characterized the sensor with a custom made cables of 10 and 15 meters long giving results similar as with short cables with no problems on the reading or noise in lab measurements. Sensor behaves according to the datasheet in the pressure range indicated by the manufacturer, two different turbo pumps have been used during the tests, a Varian TPS Compact and a Pfeiffer Hi Cube 80.

The cryopump used in the cryostat is integrated by 10 grams of Carvapur pelets ${ }^{14}$ which adsorb any outgassing and molecules during the cooling down. It is located in the back part of the LN2 tank close to the vacuum port thus when the cryostat is in the maintenance mode and under a vacuum pump the cryopump is heated to be regenerated. The cryopump allows us to have pressure below 1E-5 mbar after the LN2 transference thus improving the hold time of the cryostat.

A 10 psi safety relief valve ${ }^{15}$ is located on the back of the dewar back to maintain the integrity of the cryostat in case of an overpressure. 


\subsection{Thermal Link}

The thermal connection between the LN2 tank and the detector is possible by means of a two parts OFCH copper thermal link. One part is attached underneath the CCD mounting support and the other part is screwed directly to the copper cold finger on the LN2 tank, both parts are attached together by M3 screws which can be adjusted through a window that is located on the side of the CCD head. The geometry of the thermal link is such that it provides approximately a $0.075 \mathrm{~W} / \mathrm{K}$ thermal conductance. Despite the thermal modeling of this component the final adjustment was found experimentally so that it results in a low power heating $(0.7 \mathrm{~W}$ on the $51 \mathrm{Ohm}$ heater) to the CCD which allows regulating its operating temperature without compromising the holdtime.

\section{CRYOSTAT PERFORMANCE}

Once the cryostat is completely assembled several tests were performed to prove its behavior according to the initial requirements. Room temperature vacuum and leak checks tests of CCD head and dewar back were done independently on these subsystems to verify that the cryostat will achieve enough low pressure to work at cryogenic temperatures. Different cooling down experiments were done to experimentally investigate: LN2 hold-time, copper thermal link behavior with and without CCD, PCB equilibrium temperature, LN2 transfer time and consumption, final pressure of cryostat on cryogenic temperature. The performed tests shows that the design meets the initial requirements

\subsection{Pressure performance}

According to the design, MEGARA cryostat should have a final vacuum pressure lower or equal to 4E-6 mbar. The vacuum process is as follow: at the beginning the cryostat is at room temperature and atmospheric pressure, first is step is to pump at least to 10E-3 mbar with a mechanical pump, and after that a turbo molecular pump is activated to achieve pressures close to $1 \mathrm{E}-4$ mbar in 15 hours. Pumping the cryostat allows removing any absorbed water vapor, for that reason at the beginning of this process the activated charcoal cryopump is heated for at least 4 hours to 70-100 Celsius degrees to release any trapped molecules, this will improve its performance once its cooled to LN2 temperatures which will help to reach the final pressure and act as a backup internal pump.

Figure 4 shows a pressure curve from the starting of pumping to final pressure once the cryostat is at LN2 temperature, the change of pressure from mechanical pump to turbo pump can be seen as well as the drop in pressure when the LN2 transfer is performed. The final pressure is constant once the CCD has been tuned to its final operating temperature. Last part of the graph indicates an increase in pressure once the LN2 has boiled off from the LN2 tank. In this figure the sensor 925 MicroPirani from MKS has been used and its readout limit is at $1 \mathrm{E}-5 \mathrm{mbar}$, however in the final configuration of the cryostat the sensor 972B DualMag from MKS is used giving a final pressure of 2.8E-6 mbar once the cryostat is cold.

\subsection{Temperature performance}

The initial LN2 transfer is performed at a pressure of 5-8 psi from the storage dewar to the cryostat and the turbo pump remains active until finish the transfer. The LN2 transfer consumes 12-15 liters to fill a 6.8 liters tank in approximately 1 hour using a custom made filling tube tool that couples the cryostat and the storage dewar, the LN2 filling tube tool has two concentric tubes, the inner one allows the liquid transfer to the cryostat whilst the external one allows to release the vapor pressure to the ambient. The refills of the LN2 tank are performed in 10-15 minutes.

In figure 5, we show one complete cooling down cycle for the cryostat. The temperatures of the four cryostat sensors are shown: CCD temperature has a slow cooling down temperature which is determined by the copper thermal link, however the automatic $0.7 \mathrm{~W}$ heating power provided by the PID control of the Lakeshore 335 is activated at just below $153 \mathrm{~K}$ to regulate the temperature of the detector to its operational value; PCB temperature is tuned to $250 \mathrm{~K}$ by the careful selection of materials that support the board, this temperature allows to have the electronic components working at the temperature ranges indicated on datasheets; filling tube temperature is useful during the LN2 transfer but also to detect if there is excessive LN2 vaporization which might indicate a high pressure change in the cryostat chamber; LN2 tank temperature allows us to monitor the filling and consumption process of LN2 in the tank; finally the cryopump temperature is used only during maintenance mode to heat the charcoal and regenerate it. The cryostat behaves according to the design and has a hold time of at least 50 hours. 


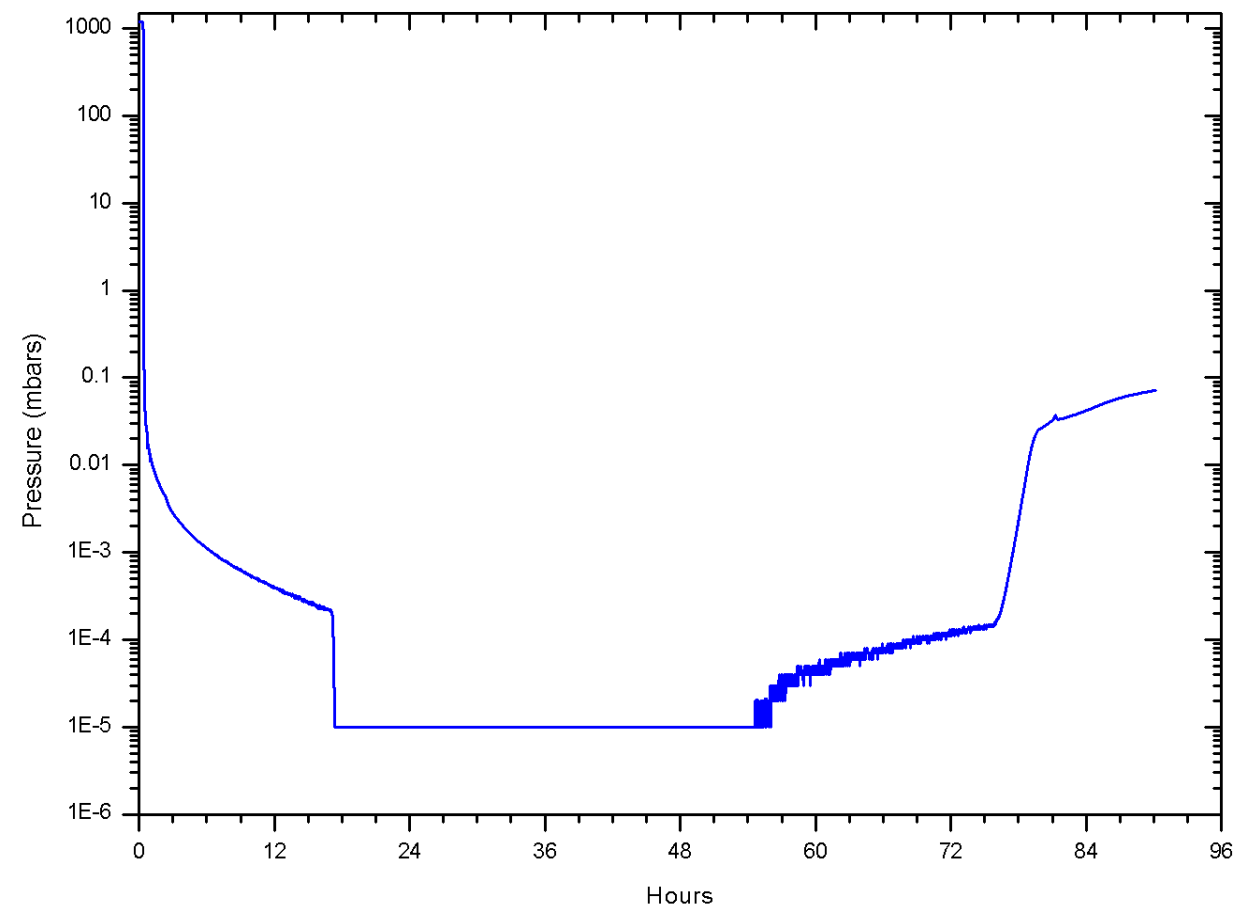

Figure 4, Pressure behavior of cryostat from room temperature and atmospheric pressure to its final stable value once the cryostat is cooled down, once the LN2 has boiled off there is a gradual increment in pressure.

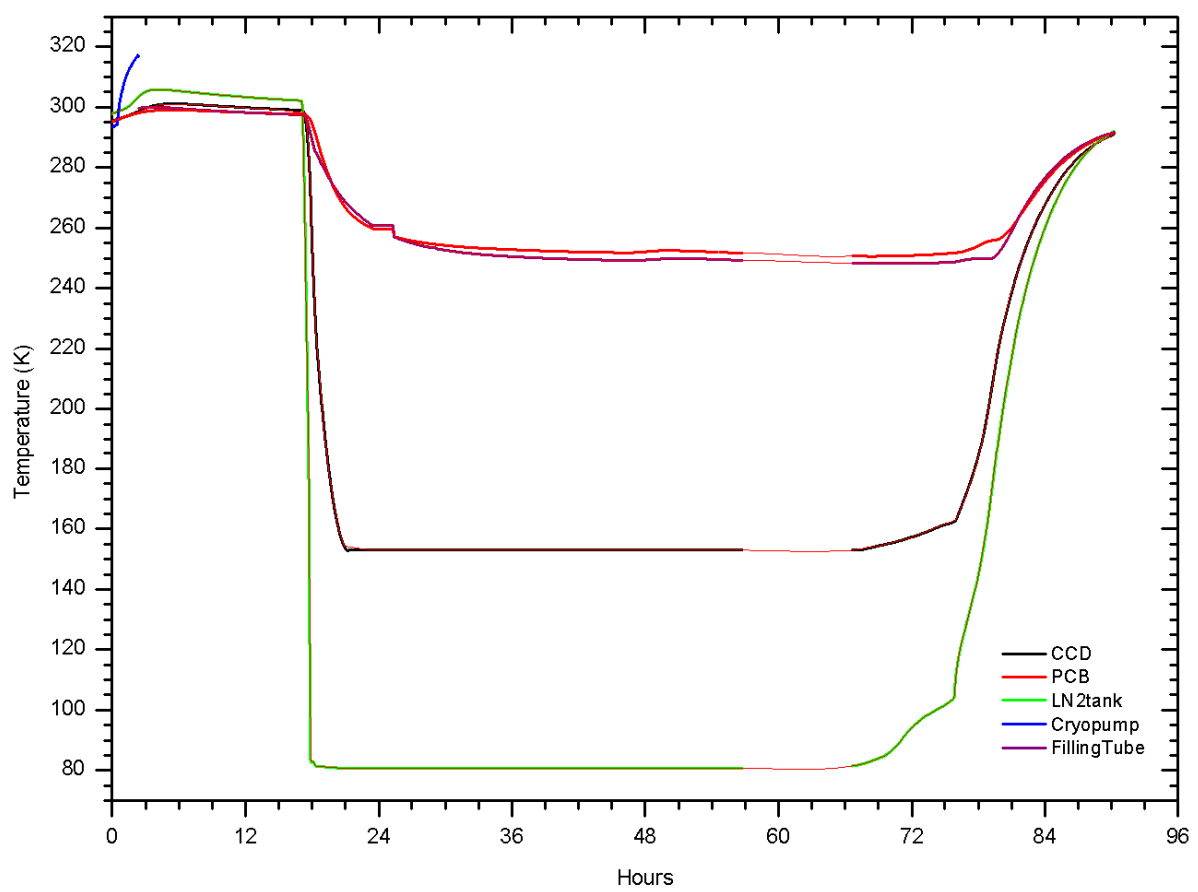

Figure 5, Megara cryostat temperature plot for one full cycle. LN2 tank sensor as expected has an abrupt drop down during the transference and then stabilizes when cryostat its full of LN2; CCD temperature has a slow cooling down determined by the copper thermal link between the tank and the CCD mounting support, final temperature of CCD is achieved by heating

power provided by the temperature controller. PCB temperature is at a safe temperature to operate the electronic components. Filling tube temperature is used during maintenance mode to regenerate the cryopump. Interpolated data has been used from hour 57 to 66 hour due to loss of data in the acquisition system. 


\section{CONCLUSIONS}

At INAOE we have successfully go through a design and AIV process for the MEGARA cryostat. The cryostat has been assembled according to the model design, dewar back and CCD-head were coupled and mounted in an optical table and it was developed a method to assembly the dewar back in vertical position which was used for transportation purposes from Mexico to Spain.

The cryostat main vacuum envelope was leak tested and was not found any problems to a level of 2E-8 mbar/lts*seg either with vacuum or sniffer methods, this condition allow us to reach pressures as low as $1 \mathrm{E}-5$ mbar at room temperature or 3E-6 mbar at LN2 temperatures, however pressures of 5E-4 mbar are good enough for LN2 transfer. Temperature sensors and vacuum gauge were also tested and characterized with their respective electronics boxes, all the signals were read without problems according to different temperature and pressure conditions to meet the initial requirements. Furthermore the CCD mounting support was positioned with micrometric screws to the nominal position to an error level below $40 \mathrm{um}$ in $\mathrm{X}, \mathrm{Y}, \mathrm{Z}$ and it was possible to provide a tilt of -0.136 degrees. The full cryostat assembly was tested for cryogenics and vacuum conditions with different optical windows, i.e. flat window, CAM-S7 dummy and real CAM-S7, no problems were found on this interface. The cryostat is able to run in cryogenic conditions with a hold time of at least 50 hours with the CCD powered and tuned to its operational temperature and an entrance optical window of $94.4 \mathrm{~mm}$ of diameter. Finally, the cryostat has been coupled to the camera assembly of the spectrograph and was cooled to provide the first images of MEGARA instrument in the lab.

\section{REFERENCES}

[1] Gil de Paz, A., et al., "MEGARA: the future optical IFU and multi-object spectrograph for the $10.4 \mathrm{~m}$ GTC telescope", Proc. of SPIE Vol. 8446, 84464Q-1-9 (2012)

[2] Gil de Paz, A., et al., "MEGARA: a new generation optical spectrograph for GTC", Proc. of SPIE, Vol. 9147, 91470O-1 (2014).

[3] Gil de Paz, A., et al., "MEGARA, the new intermediate-resolution optical IFU and MOS for GTC:

getting ready for the telescope", Proc. of SPIE, this volume, (2016)

[4] Castillo-Domínguez, E.; Ferrusca Rodríguez, D.; Tulloch, S., Velázquez, M., "Cryostat and CCD for MEGARA at GTC", Proc. of SPIE Vol. 8446, 84465Y-1-10 (2012)

[5] Ferrusca, D., et al., "MEGARA cryostat advanced design", Proc. of SPIE Vol. 9147, 91476S-1, (2014).

[6] Gil de Paz, A, et Al., "MEGARA Preliminary Design: Instrument Overview", TEC/MEG/059 2.B MEGARA consortium internal technical report, 14-18 (2012)

[7] Ferrusca, D., Castillo, E., Velázquez, M., et Al., "MEGARA Preliminary Design: Cryostat”, TEC/MEG/028 1.D MEGARA consortium internal technical report, 1-50 (2012).

[8] Tulloch, S., et Al., "MEGARA Preliminary Design: Detector and DAS", TEC/MEG/051 1.D MEGARA consortium internal technical report, 11-39 (2012).

[9] Ferrusca, D., Castillo, E., Velázquez, M., et al., "MEGARA Detailed Design: Cryostat”, TEC/MEG/104 1.A MEGARA consortium internal technical report, 1-61 (2014).

[10] Tulloch, S., et al., "MEGARA Preliminary Design: Detector and DAS", TEC/MEG/051 1.D MEGARA consortium internal technical report, 11-39 (2012)

[11] Ferrusca, D., Castillo, E., Velázquez de la Rosa, M., et al., "MEGARA Cryogenic System”, Revista Mexicana de Astronomía y Astrofísica (Serie de Conferencias) Vol. 42, 124-124 (2013)

[12] http://www.lakeshore.com/products/cryogenic-temperature-sensors/platinum-rtds/models/pages/Overview.aspx

[13] http://www.mksinst.com/product/product.aspx?ProductID=1216

[14] http://www.carbotecnia.info/

[15] http://www.mdevacuum.com/DisplayProductContent.aspx?d=MDC\&p=m.d.1.3.1 